\title{
Virtual Compton Scattering off a Spinless Target in the AdS/QCD correspondence
}

\section{Marquet}

Physics Department, Theory Unit, CERN, 1211 Genève 23, Switzerland

E-mail: cyrille.marquetecern.ch

\section{Roiesnel}

Centre de Physique Théorique, École Polytechnique, CNRS, 91128 Palaiseau, France

E-mail: Claude.Roiesnel@cpht.polytechnique.fr

\section{S. Wallon*}

LPT, Université Paris-Sud, CNRS, 91405 Orsay, France \&

UPMC Univ. Paris 06, faculté de physique, 4 place Jussieu, 75252 Paris Cedex 05, France

E-mail: wallon@th.u-psud.fr

\begin{abstract}
We perform a study of the doubly virtual Compton scattering off a spinless target $\gamma^{*} P \rightarrow \gamma^{*} P^{\prime}$ within the Anti-de Sitter(AdS)/QCD formalism. We find that the general structure allowed by the Lorentz invariance and gauge invariance of the Compton amplitude is not easily reproduced with the standard recipes of the AdS/QCD correspondence. In the soft-photon regime, where the semi-classical approximation is supposed to apply best, we show that the measurements of the electric and magnetic polarizabilities of a target like the charged pion in real Compton scattering, can already serve as stringent tests, and presumably exclude results based on the AdS/QCD correspondence in its minimal version.
\end{abstract}

35th International Conference of High Energy Physics

July 22-28, 2010

Paris, France

${ }^{*}$ Speaker. 


\section{Introduction}

The AdS/CFT correspondence [1] postulates a relation [2,3] between weakly coupled string theories living in the bulk of an anti-de Sitter (AdS) space and strongly coupled conformally invariant field theories defined on its boundary, based on the formal identification of the conformal group of the 4-d Minkowski space and of the isometry group of $\mathrm{AdS}_{5}$. The efforts within the so-called AdS/QCD approach rely on the assumption that the AdS/CFT dictionary can still describe the strong coupling regime of a confining gauge theory like QCD, despite the breaking of conformal invariance by radiative corrections.

We here report on recent results obtained in the study of the Virtual Compton scattering off a spinless target [4]. We show that the obtained amplitude does not satisfy the expected scaling for large photon virtualities. At low energy, the obtained electric and magnetic polarizabilities vanish, in contradiction with the latest experimental results.

\section{Gauge/Gravity duality}

The AdS/CFT correspondence conjectures a relation between a IIB string theory compactified on $A d S_{5} \times S^{5}$ and a $\mathscr{N}=4$ super-Yang-Mills theory. The operator in CFT $\mathscr{O}(\vec{x})$ creating asymptotic states is coupled to the source $\phi_{0}(\vec{x})$, which is the boundary value of the bulk field $\phi(\vec{x}, z)^{1}$. Both generating functions in CFT and AdS are related through:

$$
Z_{C F T}\left[\phi_{0}\right]=\left\langle e^{\int d^{4} x \phi_{0}(\vec{x}) \mathscr{O}(\vec{x})}\right\rangle_{C F T}=Z_{\text {string }}\left[\left.\phi(\vec{x}, z)\right|_{z=z_{\text {min }}}=\phi_{0}(\vec{x})\right] .
$$

The $5 \mathrm{~d}$ metric with Lorentz group $S O(1,3)$ as isometry subgroup reads (with $\eta=\operatorname{diag}(-1,1,1,1,1)$ )

$$
d s^{2}=a^{2}(z)\left(d x_{\mu} d x^{\mu}+d z^{2}\right), \quad g^{m n}=g_{m n}^{-1}=a^{-2}(z) \eta^{m n}, \quad m=0,1,2,3,4 .
$$

In the $A d S_{5}$ metric, $a(z)=R / z$ (negative curvature, corresponding to a negative cosmological constant), $\sqrt{-g}=R^{5} / z^{5}$ ( $R=1$ from now on). The UV limit $z \rightarrow z_{\min }$ is the CFT boundary. The field content is made of a $5 \mathrm{~d}$ vector $U(1)$ field $A_{m}(x, z)$, dual to the electromagnetic current, and a $5 \mathrm{~d}$ massive scalar field $\Phi(x, z)$ dual to an operator creating the spinless target.

In the large $N$ and large 't Hooft coupling $\lambda=g_{Y M}^{2} N$ limit, the IIB string theory reduces to a IIB SUGRA theory in 10 dimensions, when $R^{4} / l_{s}^{4} \sim g_{s} N \sim g_{Y M}^{2} N \gg 1, R$ being the $A d S_{5}$ and $S^{5}$ radius and $l_{s}$ the string length. In that limit $g_{s} \ll 1$ and $R \gg l_{s}$ the string theory reduces to classical SUGRA with decoupling of massive string excitations.

The conformal invariance breaking due to QCD confinement can be introduced by an IR cutoff at $z=1 / \Lambda$ [5] ("hard-wall" model) or by a background dilaton field $\chi$ [6] ("soft-wall" model), used here ${ }^{2}$. The action for $\Phi$ in this $\chi$ background reads

$$
S_{\Phi}=\frac{1}{2} \int d^{4} x d z \sqrt{-g} e^{-\chi}\left(g^{i j} \partial_{i} \Phi \partial_{j} \Phi+m_{S}^{2} \Phi^{2}\right) .
$$

The classical free field equation exhibits plane-wave solutions in $4 \mathrm{~d}$ space: $\Phi^{(0)}(x, z)=e^{i p \cdot x} \hat{\Phi}(z)$ where $\left\{\hat{\Phi}_{n}(z), n \in \mathbb{N}\right\}$ is a complete set of Kaluza-Klein (KK) solutions. The Fourier transform of

\footnotetext{
${ }^{1}$ We denote by $\vec{x}$ (or just $x$ ) the $4 \mathrm{~d}$-vector, and $z$ the holographic direction.

${ }^{2}$ Both approaches reproduce qualitatively the spectra of low lying hadron states and various decay and coupling constants [7].
} 
the obtained Green function is a bulk to bulk propagator with an explicit Källen-Lehman spectral representation when summing over the KK excitations:

$$
\widehat{G}\left(z, z^{\prime} ; p\right)=-\sum_{n=0}^{\infty} \frac{\widehat{\Phi}_{n}^{\star}(z) \widehat{\Phi}_{n}\left(z^{\prime}\right)}{p^{2}+m_{n}^{2}-i \varepsilon} .
$$

The action of a classical solution leaves only the boundary term. The classical partition function

$$
Z_{5 D}\left[\Phi_{S}\right]=\int_{\Phi\left(x, z_{\min }\right)=\Phi_{S}(x)} e^{i S[\Phi]} \propto e^{i S_{\mathrm{cl}}\left[\Phi_{S}\right]},
$$

combined with the holographic identification of two-point functions from AdS to CFT leads to

$$
\left\langle O(x) O\left(x^{\prime}\right)\right\rangle=\frac{1}{Z\left[\Phi_{S}\right]} \frac{\partial^{2} Z\left[\Phi_{S}\right]}{\partial \Phi_{S}(x) \partial \Phi_{S}\left(x^{\prime}\right)}=\frac{1}{(2 \pi)^{4}} \int_{-\infty}^{+\infty} d^{4} p e^{i p\left(x^{\prime}-x\right)} \sum_{n=0}^{\infty} a^{3}\left(z_{\min }\right) \frac{\partial_{z} \widehat{\Phi}_{n}^{\star}\left(z_{\min }\right) \widehat{\Phi}_{n}\left(z_{\min }\right)}{p^{2}+m_{n}^{2}-i \varepsilon} .
$$

From a minimal extension of the action (2.3) though a dynamical $U(1)$ bulk field coupled to $\Phi$

$$
S_{\mathrm{AdS}}\left[\Phi, \Phi^{\star}, A^{m}\right]=\int d^{4} x d z \sqrt{-g}\left(-\frac{1}{4} F^{m n} F_{m n}+e^{-\chi}\left(\left(D^{m} \Phi\right)^{\star} D_{m} \Phi+m_{S}^{2} \Phi^{\star} \Phi\right)\right),
$$

with $D_{n} \Phi=\partial_{n} \Phi-i e A_{n} \Phi$, the $n$-point correlation functions are obtained by the correspondence

$$
\begin{aligned}
Z_{\mathrm{QCD}}(c, \bar{c}, n+\bar{n}) & =\left\langle\exp \left(\int d^{4} x\left(n_{\mu}+\bar{n}_{\mu}\right) J^{\mu}+\bar{c} O+c O^{\dagger}\right)\right\rangle_{\mathrm{QCD}} \\
& =\exp \left(-S_{\mathrm{AdS}}^{c l}\left[\Phi(c), \Phi^{\star}(\bar{c}), A^{m}\left(n_{\mu}+\bar{n}_{\mu}\right)\right]\right)
\end{aligned}
$$

where $c, \bar{c}, n+\bar{n}$ are $4 \mathrm{~d}$ sources for QCD which appear as boundary conditions for the $5 \mathrm{~d}$ bulk fields. Correlation functions of QCD operators are obtained by an expansion to linear-order with respect to the sources. QCD operators are coupled to asymptotic states which are boundary conditions of normalizable bulk fields $\Phi^{(0)}, \Phi^{*(0)}$ (scalar probe). The electromagnetic current is dual to a massless non-normalizable 5d vector field $A_{m}(x, z)$ which satisfies Maxwell equation $\partial^{m}\left(z^{-1} F_{m n}\right)=0, \forall n$. Plane-wave solutions read (with the condition $\lim _{z \rightarrow 0} A(z)=1$ )

$$
A_{\mu}(x, z)=n_{\mu} e^{i q \cdot x} A(z), \quad A_{z}(x, z)=e^{i q \cdot x} A_{0}(z), \quad n^{2}=1
$$

in the Lorentz-like gauge, with $A_{0}(z)=-i(q \cdot n) / q^{2} \partial_{z} A(z)$ and $A(z)=Q z K_{1}(Q z)\left(Q^{2}=q \cdot q\right)$.

Evaluating the $\gamma^{(*)} A \rightarrow \gamma^{(*)} A^{\prime}$ Compton scattering amplitude on a spinless, or spin-averaged target requires to study a 4-point function, the first non-trivial correlator involving the propagation inside the bulk ${ }^{3}$. Based on (2.6), one should extract the coefficient of $\bar{c} n_{\mu} \bar{n}_{v} c$. This is done by solving iteratively the coupled classical equations in terms of the free bulk fields $A^{(0)}, \Phi^{*(0)}$, $\Phi^{(0)}$. One then deduces the classical action, up to $e^{2}$. The boundary condition at $z=0$ for $A^{(0)}$, $\Phi^{*(0)}, \Phi^{(0)}$ are $n_{\mu}+\bar{n}_{\mu}, c$, and $\bar{c}$, which enter in a linear way in these free fields: the $\bar{c} n_{\mu} \bar{n}_{v} c$ and $A^{(0)} A^{(0)} \Phi^{*(0)} \Phi^{(0)}$ coefficients are equal. Contracting the result on the physical plane wave boundary conditions for $A^{(0)}$ (non-normalizable), $\Phi^{*(0)}, \Phi^{(0)}$ (normalizable) gives the contributions:

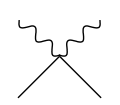

contact term

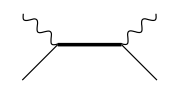

$s$-channel diagram

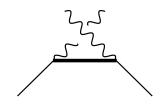

\footnotetext{
${ }^{3}$ Deep Inelastic Scattering only involves 3-point functions when evaluating matrix elements of the $U(1)$ current on a given set of intermediate states in AdS.
} 


\section{Compton scattering off an unpolarized target $A \gamma^{\star}\left(q_{1}\right)+P\left(p_{1}\right) \rightarrow \gamma^{\star}\left(q_{2}\right)+P\left(p_{2}\right)$}

Choosing $q_{1}, q_{2}$ and $p=p_{1}+p_{2}$ as the three independent momenta, the 10 independent parityconserving tensors of rank 2 reduce to 5 after implementing the electromagnetic gauge invariance:

$$
\begin{aligned}
T^{\mu v} & =V_{1}\left(g^{\mu v}-\frac{q_{1}^{\mu} q_{1}^{v}}{q_{1}^{2}}-\frac{q_{2}^{\mu} q_{2}^{v}}{q_{2}^{2}}+q_{1}^{\mu} q_{2}^{v} \frac{\left(q_{1} \cdot q_{2}\right)}{q_{1}^{2} q_{2}^{2}}\right)+V_{2}\left(p^{\mu}-q_{1}^{\mu} \frac{\left(p \cdot q_{1}\right)}{q_{1}^{2}}\right)\left(p^{v}-q_{2}^{v} \frac{\left(p \cdot q_{2}\right)}{q_{2}^{2}}\right) \\
& +V_{3}\left(q_{2}^{\mu}-q_{1}^{\mu} \frac{\left(q_{1} \cdot q_{2}\right)}{q_{1}^{2}}\right)\left(q_{1}^{v}-q_{2}^{v} \frac{\left(q_{1} \cdot q_{2}\right)}{q_{2}^{2}}\right)+V_{4}\left(p^{\mu}-q_{1}^{\mu} \frac{\left(p \cdot q_{1}\right)}{q_{1}^{2}}\right)\left(q_{1}^{v}-q_{2}^{v} \frac{\left(q_{1} \cdot q_{2}\right)}{q_{2}^{2}}\right) \\
& +V_{5}\left(q_{2}^{\mu}-q_{1}^{\mu} \frac{\left(q_{1} \cdot q_{2}\right)}{q_{1}^{2}}\right)\left(p^{v}-q_{2}^{v} \frac{\left(p \cdot q_{2}\right)}{q_{2}^{2}}\right)=\sum_{i=1}^{5} V_{i}\left(p_{1}^{2}, p_{2}^{2}, q_{1}^{2}, q_{2}^{2}, s, u\right) \mathscr{V}_{i}^{\mu v}\left(p, q_{1}, q_{2}\right) .(3.1
\end{aligned}
$$

The DVCS amplitude reduces to the Virtual Compton Scattering amplitude (VCS) when the produced photon is real, and is expressed in terms of 3 independent form factors. When additionally the incoming photon is real, the Real Compton Scattering amplitude depends only of 2 form factors.

One can now rely on the AdS/QCD correspondence to evaluate these amplitudes. In the onshell limit $\left(p_{1}^{2}=p_{2}^{2}=-m^{2}\right)$, the gauge-invariant amplitude (3.1) reduces to

$$
\begin{aligned}
T^{\mu v} & =e^{2}\left(2 \mathscr{C}_{1} \mathscr{V}_{1}^{\mu v}+\mathscr{C}_{+}\left(\mathscr{V}_{2}^{\mu v}+\mathscr{V}_{3}^{\mu v}\right)+\mathscr{C}_{-}\left(\mathscr{V}_{4}^{\mu v}+\mathscr{V}_{5}^{\mu v}\right)\right) \\
\mathscr{C}_{ \pm}\left(m^{2}, q_{1}^{2}, q_{2}^{2}, s, u\right) & =\mathscr{F}_{1}\left(m^{2}, q_{1}^{2}, q_{2}^{2}, s\right) \pm \mathscr{F}_{1}\left(m^{2}, q_{2}^{2}, q_{1}^{2}, u\right)
\end{aligned}
$$

and is thus expressed in terms of only 3 Compton form factor out of a possible 5 . The form factors $\mathscr{F}_{1}\left(m^{2}, q_{1}^{2}, q_{2}^{2}, k^{2}\right)$ and $\mathscr{C}_{1}\left(m^{2}, q_{1}^{2}, q_{2}^{2}\right)$ obtained from the AdS/QCD correspondence read (similar results were obtained within the hard wall model in Ref. [10])

$$
\begin{aligned}
\mathscr{F}_{1}\left(m^{2}, q_{1}^{2}, q_{2}^{2}, k^{2}\right) & =\iint d z_{1} d z_{2} z_{1}^{-3} e^{-\chi\left(z_{1}\right)} A_{1}\left(z_{1}\right) \widehat{\Phi}_{i}\left(z_{1}\right) \widehat{G}_{0}\left(z_{1}, z_{2}, k^{2}\right) \widehat{\Phi}_{f}^{\star}\left(z_{2}\right) A_{2}^{\star}\left(z_{2}\right) z_{2}^{-3} e^{-\chi\left(z_{2}\right)} \\
\mathscr{C}_{1}\left(m^{2}, q_{1}^{2}, q_{2}^{2}\right) & =\int d z z^{-3} e^{-\chi(z)} A_{1}(z) A_{2}^{\star}(z) \widehat{\Phi}_{i}(z) \widehat{\Phi}_{f}^{\star}(z)
\end{aligned}
$$

The $\mathscr{F}_{1}$ form factor has a natural Källen-Lehman spectral representation

$$
\begin{aligned}
\mathscr{F}_{1}\left(m^{2}, q_{1}^{2}, q_{2}^{2}, k^{2}\right) & =\sum_{n=0}^{\infty} \frac{\Gamma\left(m^{2}, m_{n}^{2}, q_{1}^{2}\right) \Gamma^{\star}\left(m^{2}, m_{n}^{2}, q_{2}^{2}\right)}{k^{2}+m_{n}^{2}-i \varepsilon}, \\
\Gamma\left(m^{2}, m_{n}^{2}, Q^{2}\right) & =Q \int d z z^{-2} e^{-\chi(z)} K_{1}(Q z) \widehat{\Phi}_{m}(z) \widehat{\Phi}_{n}^{*}(z) .
\end{aligned}
$$

The vertex function $\Gamma$ reduces to the electromagnetic form factor when $m^{2}=m_{n}^{2}$. Starting from the on-shell Compton amplitude (3.2), its absorptive part in the forward limit

$$
\left.\operatorname{Im} T^{\mu v}\left(q^{2}, s\right) \approx e^{2}\left(\frac{\partial m_{n}^{2}}{\partial n}\right)^{-1}\right|_{m_{n}^{2}=-s}\left|\Gamma\left(m^{2},-s, q^{2}\right)\right|^{2}\left(p^{\mu}+\frac{1}{x} q^{\mu}\right)\left(p^{v}+\frac{1}{x} q^{v}\right)
$$

gives the hadronic tensor of Deep Inelastic Scattering (DIS), showing that only $F_{L}$ survives, in contradiction with Callan-Gross relation. The scaling properties are governed by $\Gamma\left(m^{2}, m_{n}^{2}, Q^{2}\right)$ :

$$
\Gamma\left(m^{2}, m_{n}^{2}, Q^{2}\right) \approx \int_{0}^{1 / Q} \frac{d z}{z^{3}} e^{-\chi(z)} \widehat{\Phi}(z) \widehat{\Phi}_{n}^{*}(z) .
$$


For $Q^{2} \gg m^{2}, Q^{2} \gtrsim m_{n}^{2}$, the scaling of the hadron state $\phi(z) \underset{z \rightarrow 0}{\sim} z^{\Delta}(\Delta$ is the scaling dimension of the operator creating the spinless hadron) leads to $F_{2}\left(Q^{2}, x\right) \propto\left(1 / Q^{2}\right)^{\Delta-1}$ calling for $\Delta=1$, while for $Q^{2} \gg m^{2}, m_{n}^{2}$, the scaling properties $\phi(z) \underset{z \rightarrow 0}{\sim} z^{\Delta}$ and $\phi_{n}(z) \underset{z \rightarrow 0}{\sim} z^{\Delta}$ lead to $F_{\gamma}\left(Q^{2}\right) \propto\left(1 / Q^{2}\right)^{\Delta-1}$ calling for $\Delta=2$ [9]. This generic AdS/QCD tension due to the same scaling obtained for structure function and form factors is impossible to reconcile with a partonic picture in a simple way [8].

The VCS amplitude for $\gamma^{*} A \rightarrow \gamma A^{\prime}$ has the same tensorial structure as point-like scalar electrodynamic and depends on a single electromagnetic form factor $\mathscr{C}_{1}\left(m^{2}, q_{1}^{2}, 0\right)(3.4)$. In perturbative QCD, these form factors can in principle be related, through factorization, to generalized parton distributions (GPDs). The partonic interpretation is based on the convolution of real GPDs with coefficient functions which contain both a real and an imaginary part. In contrast, the above holographic DVCS amplitude has no absorptive part, while it is seen experimentally (through interference with the Bethe-Heitler amplitude). This may call for stringy corrections. Besides, the asymptotic behavior in $Q^{2}$ of the holographic DVCS cross-section is governed by the power-law behavior of the electromagnetic form factor, while it is expected to scale like DIS amplitude.

The Real Compton scattering $\left(q_{1}^{2}=q_{2}^{2}=0\right)$ amplitude provides informations on electric and magnetic static polarizabilities $\alpha_{E}$ and $\alpha_{M}$, defined as quadratic coefficients (in the photons energies) in corrections to Thomson scattering, defined in lab-frame $\overrightarrow{p_{1}}=\overrightarrow{0}\left(q_{i}=\omega_{i}\left(1, \widehat{q}_{i}\right), \omega_{i}^{2} \ll m_{\pi}^{2}\right)$

$$
A(\gamma \pi \rightarrow \gamma \pi)=2 e^{2} \overrightarrow{\varepsilon_{1}} \cdot \overrightarrow{\varepsilon_{2}}+8 \pi m_{\pi} \omega_{1} \omega_{2}\left(\alpha_{E} \overrightarrow{\varepsilon_{1}} \cdot \overrightarrow{\varepsilon_{2}}+\beta_{M}\left(\overrightarrow{\varepsilon_{1}} \times \widehat{q}_{1}\right) \cdot\left(\overrightarrow{\varepsilon_{2}} \times \widehat{q}_{2}\right)\right)
$$

The holographic Compton amplitude leads to vanishing static polarizabilities $\alpha_{E}$ and $\alpha_{M}$, in contradiction with the most recent experimental values for the pion [11]. One way to circumvent this problem would be to consider a non-minimal coupling between the bulk field $\Phi$ and the $U(1)$ field.

To conclude, AdS/QCD Compton amplitude is trivial in the low-energy limit. It has no partonic interpretation in the high-energy limit. Most popular AdS/QCD models incorporating flavor symmetry do not cure all the problems.

Work supported in part by the grant ANR-06-JCJC-0084.

\section{References}

[1] J.M. Malcadena, Adv. Theor. Math. Phys. 2 (1998) 231 [Int. J. Theor. Phys. 38 (1999) 1113].

[2] E. Witten, Adv. Theor. Math. Phys. 2 (1998) 253.

[3] S.S. Gubser, I.R. Klebanov and A.M. Polyakov, Phys. Lett. B428 (1998) 105.

[4] C. Marquet, C. Roiesnel and S. Wallon, JHEP 1004 (2010) 051.

[5] J. Polchinski and M.J. Strassler, Phys. Rev. Lett. 88 (2002) 031601.

[6] A. Karch, E. Katz, D.T. Son \& M.A. Stefanov, Phys. Rev. D74 (2006) 0150005.

[7] H. Boschi-Filho et al., JHEP 0305 (2003) 9; G.F. de Teramond et al., Phys. Rev. Lett. 94 (2005) 201601; T. Hambye et al., Phys. Rev. D76 (2007) 125017; P. Colangelo et al., Phys. Rev. D78 (2008) 055009.

[8] B. Pire, C. Roiesnel, L. Szymanowski and S. Wallon, Phys. Lett. B670 (2008) 84.

[9] S.J. Brodsky and G.F. de Teramond, Phys. Rev. D77 (2008) 056007.

[10] Jian-Hua Gao and Bo-Wen Xiao, Phys. Rev. D 81 (2010) 035008.

[11] Ahrens J. et al, Eur. Phys. J. A23 (2005) 113; A. Guskov [COMPASS], Fizika B 17 (2008) 313. 